OPEN ACCESS

Edited by:

Mustafa Diken,

Translational Oncology, Germany

Reviewed by:

William L. Redmond,

Earle A. Chiles Research Institute,

United States

Reno Debets,

Erasmus MC-Cancer Institute,

Netherlands

*Correspondence:

Els M. E. Verdegaal

e.m.e.verdegaal@/umc.nI

Specialty section: This article was submitted to Cancer Immunity and Immunotherapy,

a section of the journal

Frontiers in Immunology

Received: 30 June 2017

Accepted: 24 August 2017

Published: 11 September 2017

Citation:

Verdegaal EME and van der Burg SH (2017) The Potential and Challenges

of Exploiting the Vast But Dynamic

Neoepitope Landscape for

Immunotherapy.

Front. Immunol. 8:1113

doi: 10.3389/fimmu.2017.01113

\section{The Potential and Challenges of Exploiting the Vast But Dynamic Neoepitope Landscape for Immunotherapy}

\author{
Els M. E. Verdegaal* and Sjoerd H. van der Burg \\ Experimental Cancer Immunology and Therapy Group, Leiden University Medical Center, Department of Medical Oncology, \\ Leiden, Netherlands
}

Somatic non-synonymous mutations in the DNA of tumor cells may result in the presentation of tumor-specific peptides to T cells. The recognition of these so-called neoepitopes now has been firmly linked to the clinical success of checkpoint blockade and adoptive T cell therapy. Following proof-of-principle studies in preclinical models there was a surge of strategies to identify and exploit genetically defined clonally expressed neoepitopes. These approaches assume that neoepitope availability remains stable during tumor progression but tumor genetics has taught us otherwise. Under the pressure of the immune system, neoepitope expression dynamically evolves rendering neoepitope specific $T$ cells ineffective. This implies that the immunotherapeutic strategy applied should be flexible in order to cope with these changes and/or aiming at a broad range of epitopes to prevent the development of escape variants. Here, we will address the heterogeneous and dynamic expression of neoepitopes and describe our perspective and demonstrate possibilities how to further exploit the clinical potential of the neoepitope repertoire.

Keywords: somatic mutations, neoepitopes, immunotherapy, tumor heterogeneity, vaccination, adoptive cell therapy

\section{INTRODUCTION}

Spectacular progress has been made in the treatment of cancer by the introduction of checkpoint blocking antibodies against the inhibitory molecules CTLA-4, and PD-1 or its ligand PD-L1 $(1,2)$. The efficacy of these antibodies depends on the presence of antigen-specific $\mathrm{T}$ cells that can recognize tumor cells but are functionally inhibited in cancer patients (3). In melanoma (4) and lung cancer (5), clinical benefit of checkpoint blocking therapy strongly correlates with the presence of a high mutational load. This led to the hypothesis that a high number of somatic non-synonymous mutations may result in the formation of so-called neoepitopes that are recognized as truly foreign by $\mathrm{CD}^{+}$and $\mathrm{CD}^{+} \mathrm{T}$ cells, the response of which is unleashed by checkpoint blocking.

It was suggested that the clinical efficacy of adoptive cell therapy (ACT) also relies on the presence of mutation-specific $\mathrm{T}$ cells in the infusion product. Indeed, tumor infiltrating $\mathrm{T}$ cell (TIL) used for successful ACT treatment of melanoma patients (6-11), head and neck cancer (12), cholangiocarcinoma $(13,14)$, and colorectal cancer $(15)$ were shown to contain considerable frequencies of neoepitope-specific T cells. Furthermore, durable clinical responses were obtained when PBMCderived tumor-reactive $\mathrm{T}$ cells, comprising almost exclusively clonally expressed neoepitope-specific 
$\mathrm{CD}^{+}$and $\mathrm{CD}^{+} \mathrm{T}$ cells, were infused $(10,11,16)$. Furthermore, we observed that ACT products administered to responder patients contained $\mathrm{T}$ cells that recognized private rather than shared antigens as demonstrated by their almost exclusive recognition of autologous tumor cells and not a series of HLA-matched melanoma cells (Figure 1). In contrast, T cells administered to non-responders showed a broad recognition pattern. Moreover, infusion of highly enriched neoepitope-specific $\mathrm{T}$ cells resulted in clear tumor regression in a patient who relapsed after bulk TIL therapy (13). Altogether, these data suggest that approaches to select, expand and activate neoepitope specific T cells by (combinations of) checkpoint blocking, ACT and/or vaccination can improve the clinical outcome of this treatment. This, however, does not mean that we should neglect the therapeutic potential of shared tumor-antigens. This is illustrated by the complete tumor eradication of melanoma after transfer of NY-ESO-1-specific $\mathrm{CD}^{+} \mathrm{T}$ cells (17) and genetically engineered NY-ESO-1 specific T cells $(18,19)$. Although shared tumor antigens are important targets for development of immunotherapy this review focuses exclusively on the exploitation of neoepitopes.

\section{HETEROGENEITY AND DYNAMICS OF NEOEPITOPE LANDSCAPE}

Optimal exploitation of neoepitope immunity for cancer therapy requires a thorough understanding of the neoantigen landscape. Several studies have shown that the mutational landscape of a tumor is not cut into stone but dynamically evolves (20-27) with as potential outcome that tumor recognition by the immune system is lost due to reduced or lost expression of neoepitopes in recurrent tumor cell clones (11). Hence, it not only is essential to gain knowledge of the frequency and extent of intratumoral heterogeneity but also of mutational landscape changes during tumor progression and regression after treatment, including immunotherapy.

Heterogeneity of mutations occurs at spatial and temporal levels. First, different areas within a single tumor lesion may harbor different mutations. In individual tumors of eight melanoma patients, the proportion of heterogeneity of somatic mutations was reported to range from 3 to $38 \%$, although it should be mentioned that heterogeneity was particularly abundant in non-expressed genes. Nonetheless, a high degree of heterogeneity was associated with a more aggressive course of the disease (25). Second, mutations may differ between primary and metastatic lesions as well as between various metastases. Analysis of primary breast cancer lesions and matched metastases revealed that the number of genetic alterations was reduced in metastatic lesions. Although this might seem counterintuitive at first glance, it can be explained by a high grade of heterogeneous variants in the primary tumor, from which specific subclones with a less heterogeneous mutation expression but increased proliferative and metastatic potential evolve (20). Indeed, some of the mutations shared between metastatic lesions of various patients are linked to poor survival. The changes in the landscape of expressed (non-silenced) mutations vary depending on the cancer type illustrating that proportionally intratumoral heterogeneity is very high in glioma and low in NSCLC and melanoma [reviewed in Ref. (28)]. However, given the relatively high mutation rate in the latter two tumor types, the absolute number of alterations in expressed mutations is still high (28). In one exceptional case of a NSCLC patient, 99\% of the total genetic alterations (point mutations, insertions, and deletions) differed between sequential lesions (24). Finally, mutations may vary between early lesions that are sensitive to treatment and treatment-resistant recurrences. The extent of genetic alterations in these recurrent lesions varies across cancer types and is very low in ovarian cancer $(21,22)$. At the other end of the spectrum are low-grade gliomas that acquire thousands of somatic mutations that differ from the initial lesions after temozolomide therapy (23) and concomitantly evolve into a high-grade glioma phenotype. Anagnostou et al. elegantly showed that tumor lesions recurring after checkpoint blocking therapy displayed both loss and gain of putative (mutation associated) neoepitopes in four NSCLC and one HNSC patients (27). We analyzed the expression stability of six clonally expressed $\mathrm{T}$ cell targeted neoepitopes in serially obtained tumors from two stage IV melanoma patients treated by ACT (11). The data from these paired tumor samples demonstrated that under the attack of $\mathrm{T}$ cells neopitope availability was lost in four out of six cases in tumor subclones that evolved upon disease progression. These two studies show that immune pressure sculpts the mutational landscape of tumors and imply that flexibility toward the neoepitopes targeted is a prerequisite for immunotherapeutic approaches aiming to exploit the neoantigen repertoire. Recently it was reported that the number of recognized neoepitopes in TIL used for ACT of melanoma patients do not directly correlate to treatment outcome (29). There are several reasons to explain this, including the copresence of clinically active $\mathrm{T}$ cells reactive to tumor-associated antigens (17), which may have had a major contribution in the clinical responses obtained in patients who received TIL with low neoepitope-reactivity. In those patients who do not show clinical response after transfer of TIL with a high frequency of neoepitope-specific cells, a multitude of factors defined as the immunophenoscore $(30,31)$, including TME phenotype and tumor escape status, may have hampered clinical effectiveness.

Dynamics in neoantigen expression predict that strategies applying neoepitopes for reinforcement of antitumor immunity should aim at a broad panel of antigens in order to prevent escape variants. Hence, when an immunotherapeutic strategy requires epitope selection the highest priority should be given to neoepitopes derived from driver mutations. These mutations are expected to be expressed in the majority of-if not all-tumor cells and will not be lost by immunoediting because they are essential for the malignant phenotype. However, $\mathrm{T}$ cells reactive against these epitopes are infrequently detected even though several driver mutations are frequently present in various tumor types, including colorectal cancer and melanoma (15, 32, 33). Emphasis should also be given to neoepitopes derived from clonally expressed mutated genes other than acquired early during tumor evolution. In contrast to subclonal mutations, these clonal mutations may comprise driver and passenger mutations that are expressed in the "trunk" of the tumor evolutionary tree and therefore expressed in the majority of tumor cells. This notion is sustained by the observation that clinical benefit from checkpointblocking therapy is not only correlated with total tumor burden 

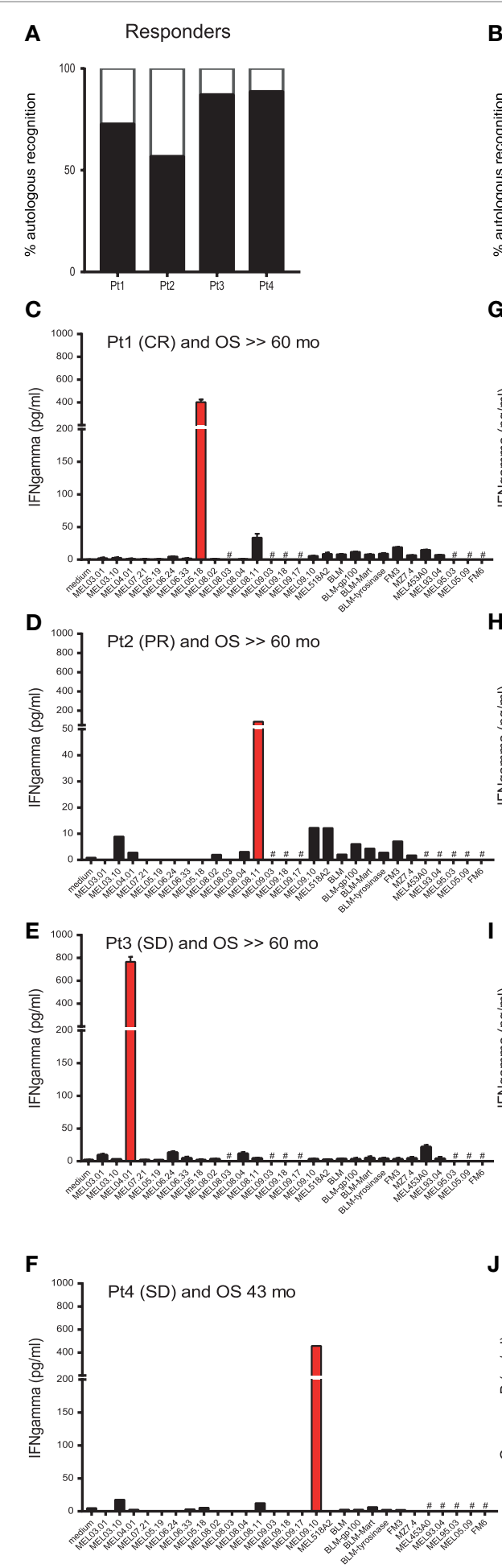
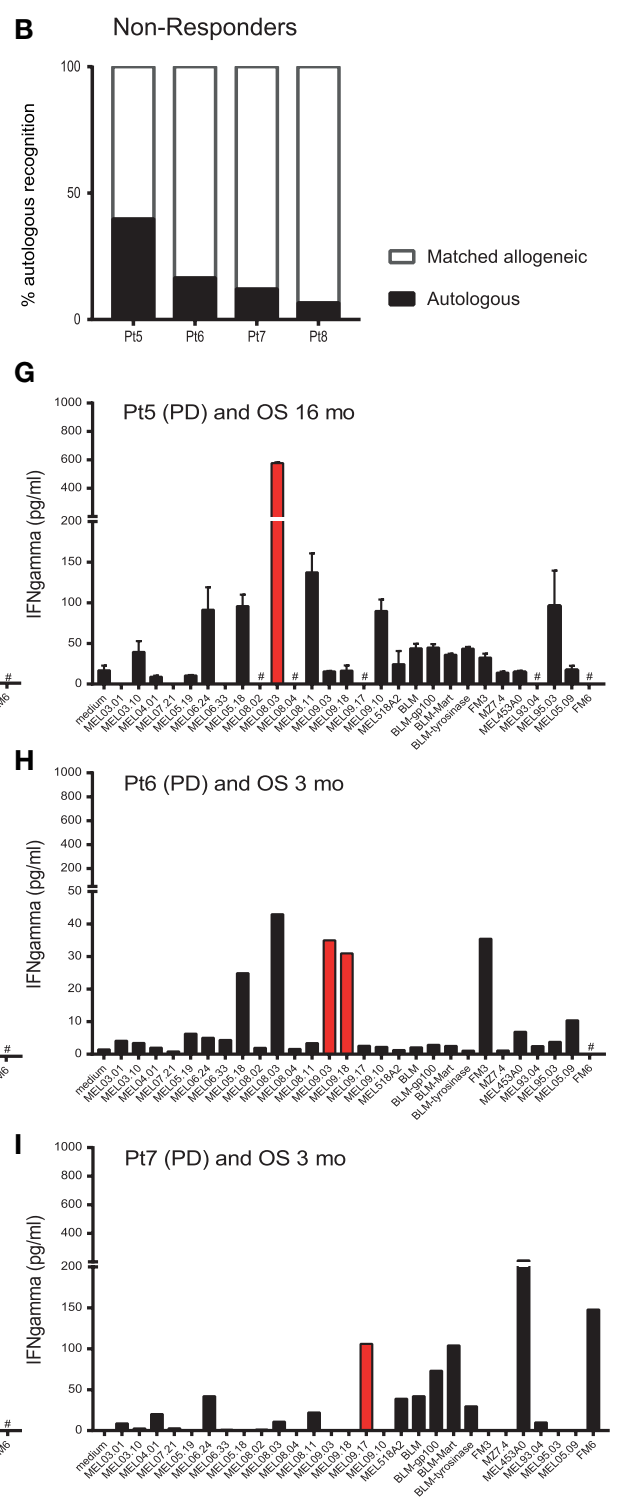

G
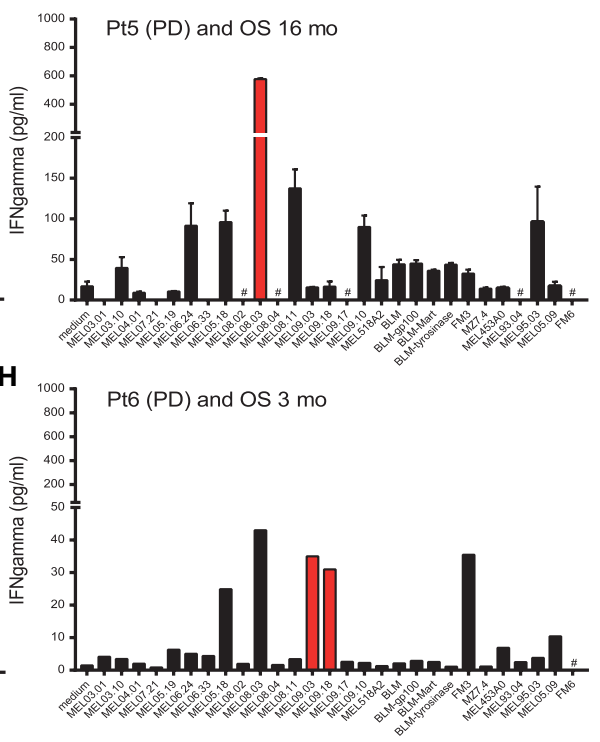

J

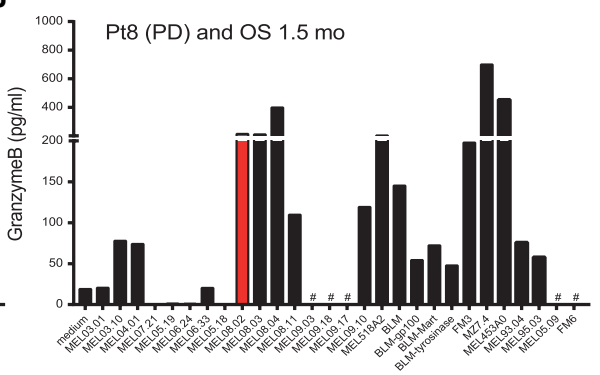

FIGURE 1 | T cell batches administered to responder patients recognize private rather than shared antigens. Tumor-reactive T cell batches were generated by repeated stimulation of PBMC with autologous melanoma cell lines in a mixed lymphocyte tumor cell culture (MLTC). These T cells were administered to melanoma patients by ACT. The patient number, with best overall response [complete response (CR), partial response (PR), stable disease (SD), or progressive disease (PD)], and overall survival (OS) in months are given. (\# = not done). IFN-gamma production, as an indicator of T cell activation, was measured after incubation of T cells used for ACT with various (partially-)matched HLA class-I melanoma cell lines. The IFN-gamma production of T cells against the autologous tumor cells is depicted as a fraction of the total IFN-gamma production against all tested cells (set at 100\%) for responder patients $(n=4)$ and non-responder patients $(n=4)$ in panels (A,B), respectively. The data of each individual patient are given in panels $(\mathbf{C}-\mathbf{J})$. Data in panels $(\mathbf{C}, \mathbf{D})$ were previously reported $(11)$. IFN-gamma production upon recognition of each cell line is represented by separate bars. The red bar in each panel indicates the autologous melanoma cell line that was used to generate the corresponding $T$ cell batch. The patients were treated in a clinical trial approved by the local ethics committee (LUMC study P04.085) and all patients gave written informed consent. 
but also correlated with homogeneity of mutations within spatial and temporally different tumor lesions in NSCLC and melanoma patients (26).

\section{EXPLOITING THE POTENTIAL OF THE VAST NUMBER OF PUTATIVE NEOEPITOPES}

The correlation between the success of checkpoint blockade and the mutational load in NSCLC, melanoma and mismatch repair deficient tumors $(4,5,34)$, demonstrates that metastasized late stage progressive cancers with concomitant high grade of intratumoral heterogeneity can be effectively targeted. It also underscores the adaptive capacity of the immune system to the dynamic mutational and neoepitope landscape. Similarly, we observed in a recurrent subclone after ACT that the expression of a non-targeted neoepitope was increased when compared to the earlier fully regressed lesions and this was paralleled by the emergence of intratumoral $\mathrm{T}$ cells specific for this neoepitope (11). However, still roughly halve of the patients do not respond to checkpoint-blocking therapy, part of which can be explained by a weak or absent pre-existing tumor-specific $\mathrm{T}$ cell response (3). Therefore, various therapeutic approaches aiming to enhance or induce (neo)antigen-specific $\mathrm{T}$ cell responses are pursued.

A logical option to harness the immune system is by identification and targeting of additional neoepitopes. So far, the number of neoepitopes eliciting a $\mathrm{T}$ cell response that are identified ranges from one to maximally ten per patient (35) and detection of neoepitope-specific T cells in ACT products or TILs has revealed that only a minority of the putative neoepitopes predicted to bind to HLA elicits spontaneous immune responses $(7,8,10,11,14$, $36,37)$. The underlying reasons are yet unknown. Most likely the selection of mutated antigens for neoepitope identification based on NGS and RNA sequencing, the prediction algorithms and $\mathrm{T}$ cell tests are far from optimal and may be improved. For instance, by more efficient capture of coding DNA regions and/or comprehensive transcriptional analysis as well as by optimization of algorithms that predict peptide processing, HLA binding, HLA-peptide stability and peptide foreignness $(38,39)$, but also by improving T-cell detection methods. A sensitive and rapid identification method to identify functional immunogenic neoepitopes is the use of DNA barcoded MHC-multimers. This allows screening of a large number of peptides in a relatively small sample of PBMC, TIL or tumor-reactive T cells (40). The sensitivity of detection may be even further enhanced when proliferation of neoepitope-specific T cells is assessed by TCR Vbeta-clonality analysis of PBMC/TIL before and after in vitro stimulation (27). The frequency of neoepitope-specific $\mathrm{T}$ cells may be low and may therefore limit detection of neoepitope immunogenicity. Selection of tumor-specific T cells from PBMC may be applied to improve outcome of functional immunogenicity tests. Actually, PD $-1^{+} \mathrm{CD} 8^{+}$and not the more abundant PD $-1^{-} \mathrm{CD} 8^{+} \mathrm{T}$ cells from peripheral blood [Figure 2B and (36)] and also from TIL (41) were shown to harbor tumor-reactive and neoepitope-specific $\mathrm{T}$ cells. Rapid identification of multiple neoepitopes per tumor sample could be readily achieved using $\mathrm{PD} 1^{+} \mathrm{CD} 8^{+}$selected TIL
(42) isolated directly ex vivo from tumor samples. It would be of interest to also investigate $\mathrm{PD}-1^{+} \mathrm{CD} 8^{+} \mathrm{T}$ cells from $\mathrm{PBMC}$ of the corresponding patients to see whether reactivity to a similar repertoire of neoepitopes is detected. Other reasons for a failure to detect more neoepitope specific $\mathrm{T}$ cells might be that spontaneously triggered neoepitope-specific $\mathrm{T}$ cells are not activated due to neoepitope heterogeneity and in particular neoepitope expression between tumor subclones (11) or because they have become exhausted or anergic (43) in TIL.

There is already some evidence that there are more neoepitopes processed and presented in the HLA molecules at the tumor cell surface than those that spontaneously raise neoepitope-specific T cell immunity. Stronen et al. showed that putative neoepitopes, not recognized by TILs, were able to trigger tumor-reactive T-cell reactivity in PBMC from healthy donors, arguing that a "neglected neoepitope repertoire" exists (37). This is also supported by the work of Carreno et al. showing that vaccination with neoepitopes that are not spontaneously recognized, does result in a putative neoepitope-specific $\mathrm{T}$ cell response in three patients with melanoma (44). Two out of seven selected immunogenic HLA- $A^{\star} 0201$-restricted neoepitopes used for vaccination of one patient, could be detected by mass spectrometry analysis to be endogenously expressed, processed and presented by HLA on tumor cells (44) and the T cells directed against these epitopes specifically lysed tumor cells expressing these two neo-antigens but not other target cells.

To gain more insight in the number of attended and neglected neoepitopes that are actually presented by HLA at the tumor cell surface mass spectrometry can be utilized. Optimal identification of neoepitopes using this approach would ideally require access to (a) a substantial amount of tumor tissue or preferentially a tumor cell line that can be cultured up to the quantities required; (b) somatic mutation data derived from sequenced exomes and transcriptome; and (c) autologous $\mathrm{T}$ cells to confirm immunogenicity of the neoepitope and functional recognition (something for which also HLA-matched naïve T cells from healthy donors can be used) as well as to show the presence of a functional T-cell repertoire in the patient, which is crucial for ultimate immune responsiveness. Identification of tumor-specific $\mathrm{T}$ cell epitopes from a fraction of tumor tissue using mass spectrometry may be limited because of the amount of available starting material for representative detection of neoepitopes among the entire HLA-ligandome (45). Nevertheless, immunogenic neoepitopes have been identified directly from melanoma biopsies (46). Mass spectrometry/ligandome data were matched with NGS/ transcriptome data for a total of five patients and led to identification of four immunogenic epitopes. In addition to the identified neoepitopes, many known and novel peptide ligands derived from tumor-associated antigens were identified, demonstrating the applicability of mass spectrometry/proteomics for broad MHC peptide ligand identification.

\section{HARNESSING THE IMMUNE SYSTEM WITH NEOEPITOPE SPECIFIC T CELLS}

From the above mentioned data, it is expected that also clinical efficacy of ACT or vaccination can be enhanced by focusing on 


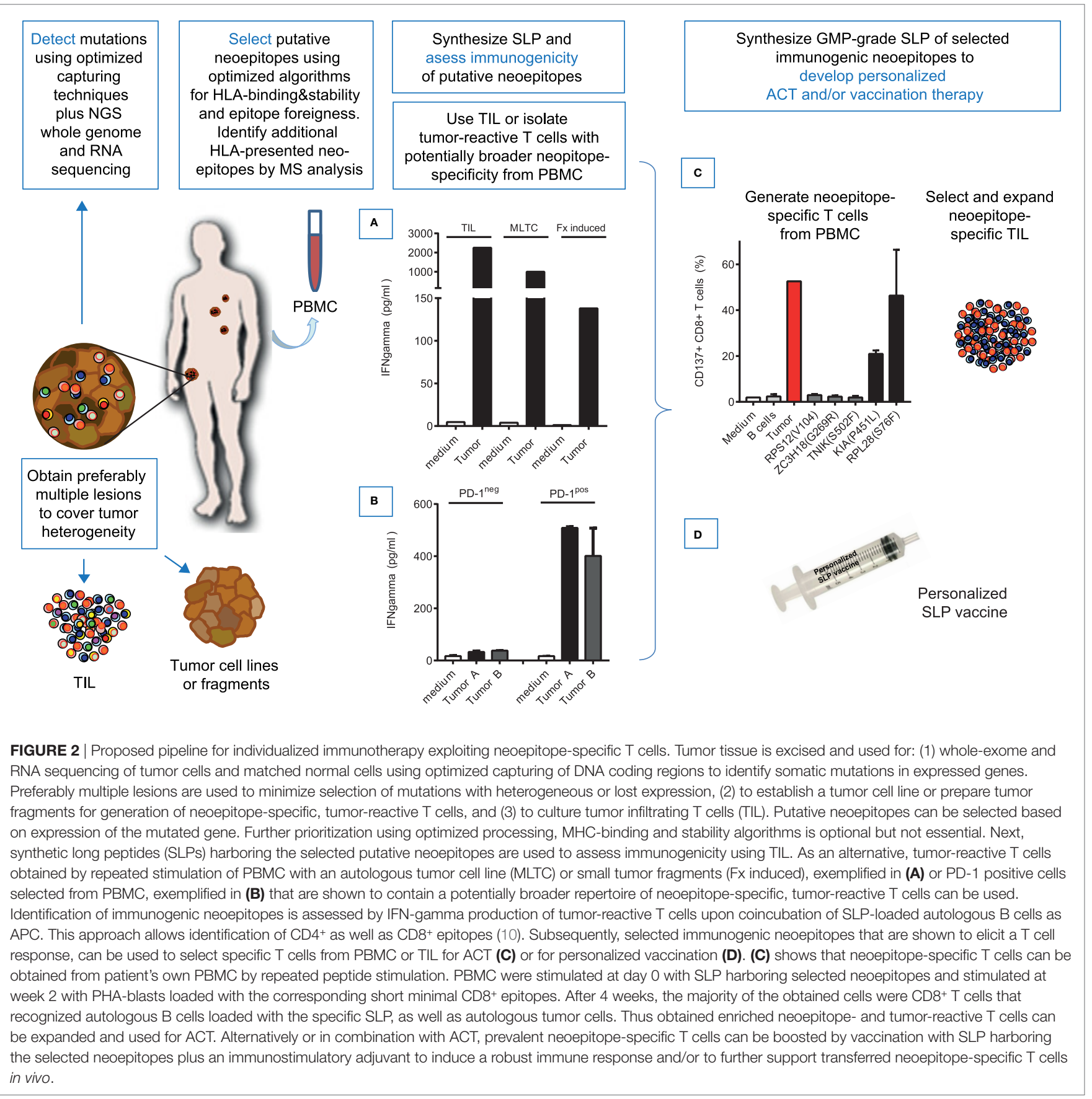

(clonally) expressed mutations-derived neoepitopes. Once a set of immunogenic neoepitopes has been identified it can be used to induce or increase the frequency of tumor-reactive $\mathrm{T}$ cells by vaccination using RNA (47), synthetic long peptides (SLPs) (48), or antigen-loaded DC (49-53). Clinical trials applying vaccination with neoepitope RNA or SLPs recently demonstrated feasibility and clinical effectiveness of neoepitope-based personalized immunotherapy $(47,48)$.

As an alternative to vaccination, selected neoepitopes can be used to expand neoepitope-specific T cells in vitro for use in ACT, for instance by stimulation of patients PBMC with SLPs covering the selected neoepitopes. We showed that SLP-stimulated T cells not only respond to neoepitope peptide-pulsed APC but also recognized autologous tumor cells, indicating that they recognize endogenously naturally presented neoepitopes (Figure 2C) and as such have clinical potential. In order to speed-up this process, one may also preselect $\mathrm{PD}-1$ positive cells from PBMC [Figure 2B and (36)] either with or without prior stimulation with autologous tumor cells or stimulation with small tumor fragments in case no autologous tumor cell line is available. 


\section{CONCLUSION AND PERSPECTIVE HOW TO EXPLOIT THE CLINICAL POTENTIAL OF THE NEOEPITOPE REPERTOIRE}

Based on the correlations between successful checkpoint therapy and mutational load as well as successful ACT and the presence of neoepitope-specific $\mathrm{T}$ cells, it is fair to assume that these neoepitope-specific $\mathrm{T}$ cells strongly contribute to the clinical effect. Clearly, the immunotherapy-mediated increased immunological pressure on the tumor in the end results in the outgrowth of tumor cell clones with downregulated or lost expression of the targeted epitopes. In most cases without direct consequences for the tumor cell itself as most of the targeted mutations are not directly involved in tumorigenesis. Importantly, the number of identified spontaneously recognized neoepitopes probably is only a fraction of the total repertoire of tumor-presented tumor-specific as well as tumor-associated antigens. To prevent neoepitope escape this broader repertoire of neoepitopes should be targeted. This, however, requires crucial improvements both with respect to the identification and the speed of the process itself. These approaches all rely on the successful identification of targetable neoepitopes, which will not be possible for all patients. In cases where no immunogenic epitopes can be identified using TIL, stimulation of PBMC with autologous tumor cells or tumor cell fragments in mixed lymphocyte tumor cell cultures (MLTCs) may result in generation of a $\mathrm{T}$ cell product enriched for tumorreactive $\mathrm{T}$ cells probably comprising considerable frequencies of undefined but effective neoepitope-specific T cells (Figure 1) with a broader neoepitope specificity when compared with TIL [Figure 2 and (54)].

\section{REFERENCES}

1. Larkin J, Hodi FS, Wolchok JD. Combined nivolumab and ipilimumab or monotherapy in untreated melanoma. N Engl J Med (2015) 373:1270-1. doi:10.1056/NEJMc1509660

2. Hodi FS, Chesney J, Pavlick AC, Robert C, Grossmann KF, McDermott DF, et al. Combined nivolumab and ipilimumab versus ipilimumab alone in patients with advanced melanoma: 2-year overall survival outcomes in a multicentre, randomised, controlled, phase 2 trial. Lancet Oncol (2016) 17:1558-68. doi:10.1016/S1470-2045(16)30366-7

3. Tumeh PC, Harview CL, Yearley JH, Shintaku IP, Taylor EJ, Robert L, et al. PD-1 blockade induces responses by inhibiting adaptive immune resistance. Nature (2014) 515:568-71. doi:10.1038/nature13954

4. Snyder A, Makarov V, Merghoub T, Yuan J, Zaretsky JM, Desrichard A, et al. Genetic basis for clinical response to CTLA-4 blockade in melanoma. N Engl J Med (2014) 371:2189-99. doi:10.1056/NEJMoa1406498

5. Rizvi NA, Hellmann MD, Snyder A, Kvistborg P, Makarov V, Havel JJ, et al. Cancer immunology. Mutational landscape determines sensitivity to PD-1 blockade in non-small cell lung cancer. Science (2015) 348:124-8. doi:10.1126/science.aaa1348

6. Lu YC, Yao X, Li YF, El-Gamil M, Dudley ME, Yang JC, et al. Mutated PPP1R3B is recognized by $T$ cells used to treat a melanoma patient who experienced a durable complete tumor regression. J Immunol (2013) 190:6034-42. doi:10.4049/jimmunol.1202830

7. van Rooij N, van Buuren MM, Philips D, Velds A, Toebes M, Heemskerk B, et al. Tumor exome analysis reveals neoantigen-specific $\mathrm{T}$-cell reactivity in an ipilimumab-responsive melanoma. J Clin Oncol (2013) 31:e439-42. doi:10.1200/JCO.2012.47.7521

8. Robbins PF, Lu YC, El-Gamil M, Li YF, Gross C, Gartner J, et al. Mining exomic sequencing data to identify mutated antigens recognized by adoptively
Assuming that a selection of immunogenic neoepitopes is available, the question remains how to optimally implicate them in effective treatment. In our opinion, the complexity of tumor biology will eventually require a combined approach to effectively combat the patient's tumor. First of all, the patient must be harnessed with tumor-reactive $\mathrm{T}$ cells, which can be accomplished by vaccination targeting neoepitopes (Figure 2D) or adoptive transfer of neoepitope specific, tumor-reactive $\mathrm{T}$ cells (Figure 2C). In addition, radiation and chemotherapy could be applied to induce tumor cell apoptosis, which can be considered as in vivo whole tumor cell vaccination, boosting the endogenous $\mathrm{T}$ cell response and stimulating antigen spreading and on top of that may promote DC trafficking and T cell priming and trafficking to non-infiltrated "cold" tumors (55-59). Moreover, chemotherapy may normalize the generally suppressive myeloid cell subsets and/or enhance the influx of potent APCs and thereby improve response to therapy (60-63). Finally, checkpoint-blocking therapy should be provided to allow optimal effector cell function of the neoepitope-specific effector $\mathrm{T}$ cells at the tumor site.

\section{AUTHOR CONTRIBUTIONS}

$\mathrm{EV}$ and $\mathrm{SB}$ designed and wrote the article.

\section{FUNDING}

This work was supported by the Dutch Cancer Society project number 10815.

transferred tumor-reactive T cells. Nat Med (2013) 19:747-52. doi:10.1038/ nm.3161

9. Lu YC, Yao X, Crystal JS, Li YF, El-Gamil M, Gross C, et al. Efficient identification of mutated cancer antigens recognized by $\mathrm{T}$ cells associated with durable tumor regressions. Clin Cancer Res (2014) 20:3401-10. doi:10.1158/10780432.CCR-14-0433

10. Linnemann C, van Buuren MM, Bies L, Verdegaal EM, Schotte R, Calis JJ, et al. High-throughput epitope discovery reveals frequent recognition of neo-antigens by CD4+ T cells in human melanoma. Nat Med (2015) 21:81-5. doi: $10.1038 / \mathrm{nm} .3773$

11. Verdegaal EM, de Miranda NF, Visser M, Harryvan T, van Buuren MM, Andersen RS, et al. Neoantigen landscape dynamics during human melanoma-T cell interactions. Nature (2016) 536:91-5. doi:10.1038/nature18945

12. Stevanovic S, Pasetto A, Helman SR, Gartner JJ, Prickett TD, Howie B, et al. Landscape of immunogenic tumor antigens in successful immunotherapy of virally induced epithelial cancer. Science (2017) 356:200-5. doi:10.1126/ science.aak 9510

13. Tran E, Turcotte S, Gros A, Robbins PF, Lu YC, Dudley ME, et al. Cancer immunotherapy based on mutation-specific CD4+ T cells in a patient with epithelial cancer. Science (2014) 344:641-5. doi:10.1126/science.1251102

14. Tran E, Ahmadzadeh M, Lu YC, Gros A, Turcotte S, Robbins PF, et al. Immunogenicity of somatic mutations in human gastrointestinal cancers. Science (2015) 350:1387-90. doi:10.1126/science.aad1253

15. Tran E, Robbins PF, Lu YC, Prickett TD, Gartner JJ, Jia L, et al. T-cell transfer therapy targeting mutant KRAS in cancer. N Engl J Med (2016) 375:2255-62. doi:10.1056/NEJMoa1609279

16. Verdegaal EM, Visser M, Ramwadhdoebe TH, van der Minne CE, van Steijn JA, Kapiteijn E, et al. Successful treatment of metastatic melanoma by adoptive transfer of blood-derived polyclonal tumor-specific CD4+ 
and CD8+ T cells in combination with low-dose interferon-alpha. Cancer Immunol Immunother (2011) 60:953-63. doi:10.1007/s00262-011-1004-8

17. Hunder NN, Wallen H, Cao J, Hendricks DW, Reilly JZ, Rodmyre R, et al. Treatment of metastatic melanoma with autologous CD4+ T cells against NY-ESO-1. N Engl J Med (2008) 358:2698-703. doi:10.1056/NEJMoa0800251

18. Robbins PF, Morgan RA, Feldman SA, Yang JC, Sherry RM, Dudley ME, et al. Tumor regression in patients with metastatic synovial cell sarcoma and melanoma using genetically engineered lymphocytes reactive with NY-ESO-1. J Clin Oncol (2011) 29:917-24. doi:10.1200/JCO.2010.32.2537

19. Robbins PF, Kassim SH, Tran TL, Crystal JS, Morgan RA, Feldman SA, et al. A pilot trial using lymphocytes genetically engineered with an NY-ESO-1reactive T-cell receptor: long-term follow-up and correlates with response. Clin Cancer Res (2015) 21:1019-27. doi:10.1158/1078-0432.CCR-14-2708

20. Poplawski AB, Jankowski M, Erickson SW, Diaz de ST, Partridge EC, Crasto $\mathrm{C}$, et al. Frequent genetic differences between matched primary and metastatic breast cancer provide an approach to identification of biomarkers for disease progression. Eur J Hum Genet (2010) 18:560-8. doi:10.1038/ ejhg.2009.230

21. Castellarin M, Milne K, Zeng T, Tse K, Mayo M, Zhao Y, et al. Clonal evolution of high-grade serous ovarian carcinoma from primary to recurrent disease. J Pathol (2013) 229:515-24. doi:10.1002/path.4105

22. Wick DA, Webb JR, Nielsen JS, Martin SD, Kroeger DR, Milne K, et al. Surveillance of the tumor mutanome by $\mathrm{T}$ cells during progression from primary to recurrent ovarian cancer. Clin Cancer Res (2014) 20:1125-34. doi:10.1158/1078-0432.CCR-13-2147

23. Johnson BE, Mazor T, Hong C, Barnes M, Aihara K, McLean CY, et al. Mutational analysis reveals the origin and therapy-driven evolution of recurrent glioma. Science (2014) 343:189-93. doi:10.1126/science.1239947

24. Biswas R, Gao S, Cultraro CM, Maity TK, Venugopalan A, Abdullaev Z, et al. Genomic profiling of multiple sequentially acquired tumor metastatic sites from an "exceptional responder" lung adenocarcinoma patient reveals extensive genomic heterogeneity and novel somatic variants driving treatment response. Cold Spring Harb Mol Case Stud (2016) 2:a001263. doi:10.1101/mcs. a001263

25. Harbst K, Lauss M, Cirenajwis H, Isaksson K, Rosengren F, Torngren T, et al. Multiregion whole-exome sequencing uncovers the genetic evolution and mutational heterogeneity of early-stage metastatic melanoma. Cancer Res (2016) 76:4765-74. doi:10.1158/0008-5472.CAN-15-3476

26. McGranahan N, Furness AJ, Rosenthal R, Ramskov S, Lyngaa R, Saini SK, et al. Clonal neoantigens elicit $\mathrm{T}$ cell immunoreactivity and sensitivity to immune checkpoint blockade. Science (2016) 351:1463-9. doi:10.1126/science.aaf1490

27. Anagnostou V, Smith KN, Forde PM, Niknafs N, Bhattacharya R, White J, et al. Evolution of neoantigen landscape during immune checkpoint blockade in non-small cell lung cancer. Cancer Discov (2017) 7:264-76. doi:10.1158/21598290.CD-16-0828

28. McGranahan N, Swanton C. Clonal heterogeneity and tumor evolution: past, present, and the future. Cell (2017) 168:613-28. doi:10.1016/j.cell.2017.01.018

29. Robbins PF. Tumor-infiltrating lymphocyte therapy and neoantigens. Cancer $J$ (2017) 23:138-43. doi:10.1097/PPO.0000000000000267

30. Angelova M, Charoentong P, Hackl H, Fischer ML, Snajder R, Krogsdam AM, et al. Characterization of the immunophenotypes and antigenomes of colorectal cancers reveals distinct tumor escape mechanisms and novel targets for immunotherapy. Genome Biol (2015) 16:64. doi:10.1186/s13059-015-0620-6

31. Charoentong P, Finotello F, Angelova M, Mayer C, Efremova M, Rieder D, et al. Pan-cancer immunogenomic analyses reveal genotype-immunophenotype relationships and predictors of response to checkpoint blockade. Cell Rep (2017) 18:248-62. doi:10.1016/j.celrep.2016.12.019

32. Sharkey MS, Lizee G, Gonzales MI, Patel S, Topalian SL. CD4(+) T-cell recognition of mutated B-RAF in melanoma patients harboring the V599E mutation. Cancer Res (2004) 64:1595-9. doi:10.1158/0008-5472.CAN-03-3231

33. Somasundaram R, Swoboda R, Caputo L, Otvos L, Weber B, Volpe P, et al. Human leukocyte antigen-A2-restricted CTL responses to mutated BRAF peptides in melanoma patients. Cancer Res (2006) 66:3287-93. doi:10.1158/0008-5472.CAN-05-1932

34. Le DT, Uram JN, Wang H, Bartlett BR, Kemberling H, Eyring AD, et al. PD-1 blockade in tumors with mismatch-repair deficiency. N Engl J Med (2015) 372:2509-20. doi:10.1056/NEJMoa1500596

35. Prickett TD, Crystal JS, Cohen CJ, Pasetto A, Parkhurst MR, Gartner JJ, et al. Durable complete response from metastatic melanoma after transfer of autologous $\mathrm{T}$ cells recognizing 10 mutated tumor antigens. Cancer Immunol Res (2016) 4:669-78. doi:10.1158/2326-6066.CIR-15-0215

36. Gros A, Parkhurst MR, Tran E, Pasetto A, Robbins PF, Ilyas S, et al. Prospective identification of neoantigen-specific lymphocytes in the peripheral blood of melanoma patients. Nat Med (2016) 22:433-8. doi:10.1038/nm.4051

37. Stronen E, Toebes M, Kelderman S, van Buuren MM, Yang W, van RN, et al. Targeting of cancer neoantigens with donor-derived $\mathrm{T}$ cell receptor repertoires. Science (2016) 352:1337-41. doi:10.1126/science.aaf2288

38. Yarchoan M, Johnson BA III, Lutz ER, Laheru DA, Jaffee EM. Targeting neoantigens to augment antitumour immunity. Nat Rev Cancer (2017) 17:209-22. doi:10.1038/nrc.2016.154

39. Tureci O, Vormehr M, Diken M, Kreiter S, Huber C, Sahin U. Targeting the heterogeneity of cancer with individualized neoepitope vaccines. Clin Cancer Res (2016) 22:1885-96. doi:10.1158/1078-0432.CCR-15-1509

40. Bentzen AK, Marquard AM, Lyngaa R, Saini SK, Ramskov S, Donia M, et al. Large-scale detection of antigen-specific T cells using peptide-MHC-I multimers labeled with DNA barcodes. Nat Biotechnol (2016) 34:1037-45. doi:10.1038/nbt.3662

41. Gros A, Robbins PF, Yao X, Li YF, Turcotte S, Tran E, et al. PD-1 identifies the patient-specific $\mathrm{CD} 8(+)$ tumor-reactive repertoire infiltrating human tumors. J Clin Invest (2014) 124:2246-59. doi:10.1172/JCI73639

42. Pasetto A, Gros A, Robbins PF, Deniger DC, Prickett TD, Matus-Nicodemos R, et al. Tumor- and neoantigen-reactive T-cell receptors can be identified based on their frequency in fresh tumor. Cancer Immunol Res (2016) 4:734-43. doi:10.1158/2326-6066.CIR-16-0001

43. Zarour HM. Reversing T-cell dysfunction and exhaustion in cancer. Clin Cancer Res (2016) 22:1856-64. doi:10.1158/1078-0432.CCR-15-1849

44. Carreno BM, Magrini V, Becker-Hapak M, Kaabinejadian S, Hundal J, Petti AA, et al. A dendritic cell vaccine increases the breadth and diversity of melanoma neoantigen-specific T cells. Science (2015) 348:803-8. doi:10.1126/science. aaa3828

45. Polyakova A, Kuznetsova K, Moshkovskii S. Proteogenomics meets cancer immunology: mass spectrometric discovery and analysis of neoantigens. Expert Rev Proteomics (2015) 12:533-41. doi:10.1586/14789450.2015.1070100

46. Bassani-Sternberg M, Braunlein E, Klar R, Engleitner T, Sinitcyn P, Audehm S, et al. Direct identification of clinically relevant neoepitopes presented on native human melanoma tissue by mass spectrometry. Nat Commun (2016) 7:13404. doi:10.1038/ncomms13404

47. Sahin U, Derhovanessian E, Miller M, Kloke BP, Simon P, Lower M, et al. Personalized RNA mutanome vaccines mobilize poly-specific therapeutic immunity against cancer. Nature (2017) 547:222-6. doi:10.1038/nature23003

48. Ott PA, Hu Z, Keskin DB, Shukla SA, Sun J, Bozym DJ, et al. An immunogenic personal neoantigen vaccine for patients with melanoma. Nature (2017) 547:217-21. doi:10.1038/nature22991

49. Kreiter S, Vormehr M, van de Roemer N, Diken M, Lower M, Diekmann J, et al. Mutant MHC class II epitopes drive therapeutic immune responses to cancer. Nature (2015) 520:692-6. doi:10.1038/nature14426

50. Grabbe S, Haas H, Diken M, Kranz LM, Langguth P, Sahin U. Translating nanoparticulate-personalized cancer vaccines into clinical applications: case study with RNA-lipoplexes for the treatment of melanoma. Nanomedicine (Lond) (2016) 11:2723-34. doi:10.2217/nnm-2016-0275

51. Diken M, Kranz LM, Kreiter S, Sahin U. mRNA: a versatile molecule for cancer vaccines. Curr Issues Mol Biol (2017) 22:113-28. doi:10.21775/cimb.022.113

52. Gubin MM, Zhang X, Schuster H, Caron E, Ward JP, Noguchi T, et al. Checkpoint blockade cancer immunotherapy targets tumour-specific mutant antigens. Nature (2014) 515:577-81. doi:10.1038/nature13988

53. Schumacher T, Bunse L, Pusch S, Sahm F, Wiestler B, Quandt J, et al. A vaccine targeting mutant IDH1 induces antitumour immunity. Nature (2014) 512:324-7. doi:10.1038/nature13387

54. Verdegaal EM. Adoptive cell therapy: a highly successful individualized therapy for melanoma with great potential for other malignancies. Curr Opin Immunol (2016) 39:90-5. doi:10.1016/j.coi.2016.01.004

55. Ma Y, Adjemian S, Mattarollo SR, Yamazaki T, Aymeric L, Yang H, et al. Anticancer chemotherapy-induced intratumoral recruitment and differentiation of antigen-presenting cells. Immunity (2013) 38:729-41. doi:10.1016/j. immuni.2013.03.003

56. Kroemer G, Galluzzi L, Kepp O, Zitvogel L. Immunogenic cell death in cancer therapy. Annu Rev Immunol (2013) 31:51-72. doi:10.1146/ annurev-immunol-032712-100008 
57. Ahmed KA, Kim S, Arrington J, Naghavi AO, Dilling TJ, Creelan BC, et al. Outcomes targeting the PD-1/PD-L1 axis in conjunction with stereotactic radiation for patients with non-small cell lung cancer brain metastases. J Neurooncol (2017) 133:331-8. doi:10.1007/s11060-017-2437-5

58. Ahmed KA, Abuodeh YA, Echevarria MI, Arrington JA, Stallworth DG, Hogue $\mathrm{C}$, et al. Clinical outcomes of melanoma brain metastases treated with stereotactic radiosurgery and anti-PD-1 therapy, anti-CTLA-4 therapy, BRAF/ MEK inhibitors, BRAF inhibitor, or conventional chemotherapy. Ann Oncol (2016) 27:2288-94. doi:10.1093/annonc/mdw417

59. Dovedi SJ, Cheadle EJ, Popple AL, Poon E, Morrow M, Stewart R, et al. Fractionated radiation therapy stimulates antitumor immunity mediated by both resident and infiltrating polyclonal T-cell populations when combined with PD-1 blockade. Clin Cancer Res (2017). doi:10.1158/1078-0432.CCR-16-1673

60. Zitvogel L, Kepp O, Kroemer G. Immune parameters affecting the efficacy of chemotherapeutic regimens. Nat Rev Clin Oncol (2011) 8:151-60. doi:10.1038/ nrclinonc.2010.223

61. van Meir H, Kenter GG, Burggraaf J, Kroep JR, Welters MJ, Melief CJ, et al. The need for improvement of the treatment of advanced and metastatic cervical cancer, the rationale for combined chemo-immunotherapy. Anticancer Agents Med Chem (2014) 14:190-203. doi:10.2174/18715206113136660372
62. Welters MJ, van der Sluis TC, van MH, Loof NM, van Ham VJ, van DS, et al. Vaccination during myeloid cell depletion by cancer chemotherapy fosters robust T cell responses. Sci Transl Med (2016) 8:334ra52. doi:10.1126/ scitranslmed.aad8307

63. Beyranvand NE, van der Sluis TC, van DS, Yagita H, Janssen GM, van Veelen PA, et al. Tumor eradication by cisplatin is sustained by CD80/86mediated costimulation of CD8+ T cells. Cancer Res (2016) 76:6017-29. doi:10.1158/0008-5472.CAN-16-0881

Conflict of Interest Statement: The authors declare that the research was conducted in the absence of any commercial or financial relationships that could be construed as a potential conflict of interest.

Copyright (c) 2017 Verdegaal and van der Burg. This is an open-access article distributed under the terms of the Creative Commons Attribution License (CC BY). The use, distribution or reproduction in other forums is permitted, provided the original author(s) or licensor are credited and that the original publication in this journal is cited, in accordance with accepted academic practice. No use, distribution or reproduction is permitted which does not comply with these terms. 